\title{
Theoretical insights into the molecular mechanism of I117V mutation in neuraminidase mediated reduction of oseltamivir drug susceptibility in A/H5N1 influenza virus
}

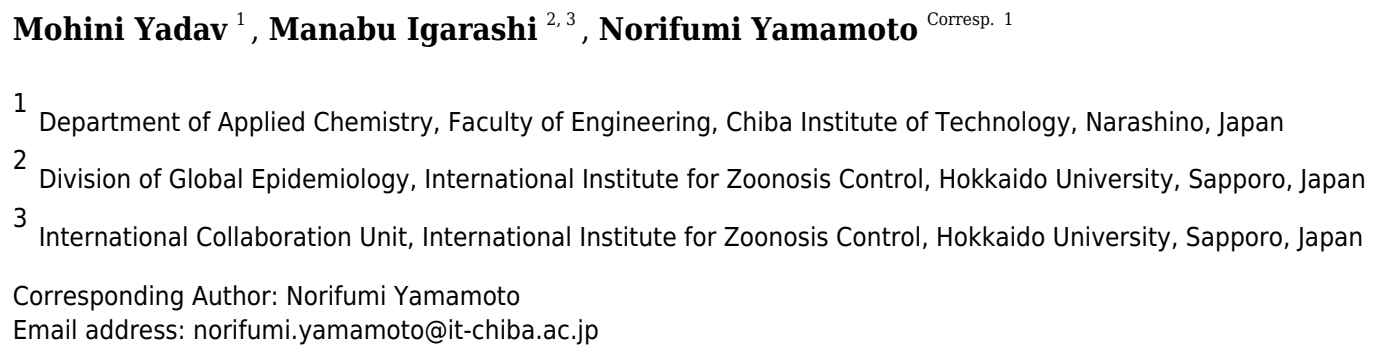

The substitution of lle to Val at residue 117 (I117V) of neuraminidase (NA) reduces the susceptibility of the A/H5N1 influenza virus to oseltamivir (OTV). However, the molecular mechanism by which the I117V mutation affects the intermolecular interactions between NA and OTV has not been fully elucidated. In this study, we performed molecular dynamics (MD) simulations to analyze the characteristic conformational changes that contribute to the reduced binding affinity of NA to OTV after the I117V mutation. The results of MD simulations revealed that after the I117V mutation in NA, the changes in the secondary structure around the mutation site had a noticeable effect on the residue interactions in the OTV-binding site. In the case of the WT NA-OTV complex, the positively charged side chain of R118, located in the $\beta$-sheet region, frequently interacted with the negatively charged side chain of E119, which is an amino acid residue in the OTV-binding site. This can reduce the electrostatic repulsion of E119 toward D151, which is also a negatively charged residue in the OTV-binding site, so that both E119 and D151 simultaneously form hydrogen bonds with OTV more frequently, which greatly contributes to the binding affinity of NA to OTV. After the I117V mutation in NA, the side chain of R118 interacted with the side chain of E119 less frequently, likely because of the decreased tendency of R118 to form a $\beta$-sheet structure. As a result, the electrostatic repulsion of E119 toward D151 is greater than that of the WT case, making it difficult for both E119 and D151 to simultaneously form hydrogen bonds with OTV, which in turn reduces the binding affinity of NA to OTV. Hence, after the I117V mutation in NA, influenza viruses are less susceptible to OTV because of conformational changes in residues of R118, E119, and D151 around the mutation site and in the binding site. 
1 Theoretical insights into the molecular mechanism of

2 I117V mutation in neuraminidase mediated reduction

3 of oseltamivir drug susceptibility in A/H5N1 influenza

\section{4 virus}

5

$6{\text { Mohini } \text { Yadav }^{1} \text {, Manabu Igarashi }}^{2,3}$, Norifumi Yamamoto ${ }^{1}$

7

$8{ }^{1}$ Department of Applied Chemistry, Faculty of Engineering, Chiba Institute of Technology,

9 Narashino, Japan

$10{ }^{2}$ Division of Global Epidemiology, International Institute for Zoonosis Control, Hokkaido

11 University, Sapporo, Japan

$12{ }^{3}$ International Collaboration Unit, International Institute for Zoonosis Control, Hokkaido

13 University, Sapporo, Japan

14

15 Corresponding Author:

16 Norifumi Yamamoto ${ }^{1}$

17 Tsudanuma 2-17-1, Narashino, Chiba 275-0016, Japan

18 Email address: norifumi.yamamoto@it-chiba.ac.jp 


\section{Abstract}

22 The substitution of Ile to Val at residue 117 (I117V) of neuraminidase (NA) reduces the 23 susceptibility of the A/H5N1 influenza virus to oseltamivir (OTV). However, the molecular 24 mechanism by which the I117V mutation affects the intermolecular interactions between NA and 25 OTV has not been fully elucidated. In this study, we performed molecular dynamics (MD) 26 simulations to analyze the characteristic conformational changes that contribute to the reduced 27 binding affinity of NA to OTV after the I117V mutation. The results of MD simulations revealed 28 that after the I117V mutation in NA, the changes in the secondary structure around the mutation 29 site had a noticeable effect on the residue interactions in the OTV-binding site. In the case of the 30 WT NA-OTV complex, the positively charged side chain of R118, located in the $\beta$-sheet region, 31 frequently interacted with the negatively charged side chain of E119, which is an amino acid 32 residue in the OTV-binding site. This can reduce the electrostatic repulsion of E119 toward 33 D151, which is also a negatively charged residue in the OTV-binding site, so that both E119 and 34 D151 simultaneously form hydrogen bonds with OTV more frequently, which greatly 35 contributes to the binding affinity of NA to OTV. After the I117V mutation in NA, the side chain 36 of R118 interacted with the side chain of E119 less frequently, likely because of the decreased 37 tendency of R118 to form a $\beta$-sheet structure. As a result, the electrostatic repulsion of E119 38 toward D151 is greater than that of the WT case, making it difficult for both E119 and D151 to 39 simultaneously form hydrogen bonds with OTV, which in turn reduces the binding affinity of 40 NA to OTV. Hence, after the I117V mutation in NA, influenza viruses are less susceptible to 41 OTV because of conformational changes in residues of R118, E119, and D151 around the 42 mutation site and in the binding site. 


\section{Introduction}

Influenza A viruses infect a variety of avian and mammalian species, including humans (Webster et al., 1992). Influenza A viruses are divided into subtypes based on antigenic differences of two virus surface glycoproteins, hemagglutinin (HA) and neuraminidase (NA) (Gamblin \& Skehel, 2010). Sixteen HA (H1-H16) and nine NA (N1-N9) subtypes have been isolated from wild waterfowl so far (Fouchier et al., 2005). HA mediates virus entry into the host cell by binding to a terminal sialic acid on the host cell surface. NA is responsible for removing sialic acid to facilitate the release of progeny viruses from infected cells. Several NA inhibitors, such as oseltamivir (OTV), zanamivir, laninamivir, and peramivir, are currently available for the treatment of influenza virus infection (McKimm-Breschkin, 2012). Among them, OTV is the most widely used anti-influenza drug (Kim et al., 1997).

OTV-resistant H1N1 and H5N1 viruses have been isolated from humans as well as avian or swine species (Monto et al., 2006; Rameix-Welti et al., 2006; McKimm-Breschkin et al., 2007). This suggests that viruses could acquire reduced sensitivity to OTV not only by drugselective pressure but also by natural genetic variation. In the mid-2000s, several H5N1 viruses with an Ile-to-Val substitution at position 117 of NA (I117V) were isolated from some avian species (Hurt et al., 2007; McKimm-Breschkin et al., 2007; Govorkova et al., 2009; Chen et al., 2010; Ilyushina et al., 2010; McKimm-Breschkin et al., 2013; Takano et al., 2013; MarinovaPetkova et al., 2014; Creanga et al., 2017; Kode et al., 2019). In vitro and in vivo experiments have shown that the I117V mutant NA conferred a reduction in susceptibility to OTV as compared to the wild-type (WT) (Hurt et al., 2007; McKimm-Breschkin et al., 2007; Chen et al., 2010; Ilyushina et al., 2010; McKimm-Breschkin et al., 2013; Takano et al., 2013; Creanga et al., 2017; Kode et al., 2019). Interestingly, residue 117 is not contained in the drug-binding site of NA, which consists of eight functional residues (R118, D151, R152, R224, E276, R292, R371, and Y406; N2 numbering) and eleven framework residues (E119, R156, W178, S179, D198, I222, E227, H274, E277, N294, and E425; N2 numbering) (Colman, Varghese \& Laver, 1983; Colman, Hoyne \& Lawrence, 1993). The molecular mechanism underlying how the mutation of residue 117 , which is not part of the drug-binding site of NA, indirectly affects the molecular interaction between NA and OTV has not been fully elucidated.

Several computational studies using molecular dynamics (MD) simulations have reported on the molecular mechanism of reduced susceptibility to OTV in the I117V mutant (Takano et al., 2013; Mhlongo \& Soliman, 2015). Takano et al. (2013) evaluated the effects of the I117V mutation in NA on OTV susceptibility in vitro, in vivo, and in silico. Their experimental results showed that the I117V mutation caused a slight reduction in the susceptibility of NA to OTV in vitro and dramatically in vivo. They also analyzed a single 2.5 -ns trajectory obtained from MD simulations to further investigate the molecular mechanism by which the I117V mutation reduces the susceptibility of NA to OTV. Their computational results showed that the I117V mutation decreased the binding affinity for OTV because of the loss of hydrogen bonds between the carboxyl group of OTV and the side chain of R118 of NA. Mhlongo and Soliman (2015) analyzed four distinctive 25-ns trajectories obtained from MD simulations to investigate the molecular mechanism of the reduced susceptibility of the I117V mutant NA to OTV. Their computational results showed that the I117V mutation distorts the orientation of OTV in the drug-binding site of NA because of the loss of hydrogen bonds between the amino group of OTV and the side chain of E1 19 of NA, resulting in reduced binding affinity of NA to OTV. In these previous computational studies, the production trajectories of the MD simulations were too short to reach reliable statistical results. In addition, they focused on changes in the direct interactions 
91 between OTV and amino acid residues in the drug-binding site of NA. However, it was not clear

92 how the I117V mutation of NA at a point outside its drug-binding site could cause changes in the

93 intermolecular interaction with OTV.

In this study, we performed four distinctive 100-ns MD simulations for the WT and I117V mutant NA-OTV complexes in the A/H5N1 influenza virus. Based on the multiple production trajectories obtained from MD simulations, we analyzed the characteristic conformational changes around the I117V mutation site of NA, which greatly affected the intermolecular interactions with OTV. The results showed that after the I117V mutation in NA, the binding affinity between NA and OTV was reduced due to the conformational change of R118 adjacent to the mutation site, which affected the interactions of E119 and D151 with OTV. Thus, the present study successfully clarified the molecular mechanism by which the I117V mutation reduces the susceptibility of NA to OTV.

103

104

105

\section{Methods}

\section{Initial structures}

The coordinate of WT avian influenza virus A/H5N1 NA in complex with OTV was obtained from the Protein Data Bank (PDB code: 2HU4) (Russell et al., 2006). The complex structure of the I117V mutant NA and OTV was generated by replacing isoleucine (Ile) 117 in the WT complex with valine (Val). H5N1 NA contains one calcium ion, which is necessary for structural stability (Smith et al., 2006), but no calcium ions were found in the crystal structure registered as 2HU4. The coordinate of the calcium ion in NA was obtained from the structure of A/H5N1 NA registered as 3CL0 (Collins et al., 2008). The protonation state of histidine (His) in NA at pH 7 was determined using the PDB2PQR server (Dolinsky et al., 2004). The other ionized residues,

114 such as arginine (Arg), lysine (Lys), aspartic acid (Asp), and glutamic acid (Glu), were treated as charged entities. The missing hydrogen atoms in NA and OTV were added using the LEaP program in the Amber 20 package (Case et al., 2020). For each disulfide bond in NA, a covalent bond was created between the proximate cysteine residues using the LEaP program. The FF14SB variant of the AMBER force field was used to describe NA (Maier et al., 2015). The parameters of the generalized AMBER force field (GAFF) were applied to OTV (Wang et al., 2004). The partial atomic charges in OTV were determined on the basis of ab initio quantum chemistry calculations at the HF/6-31G(d) level with the Gaussian 16 program package (Frisch et al., 2016), following the restrained electrostatic potential fitting procedure (Bayly et al., 1993). The complexes of NA and OTV were dissolved in a truncated octahedral box filled with water molecules, where the box size was set so that there was a distance of at least $10 \AA$ between the complexes and the boundary of the box. The TIP3P model was used to represent water molecules (Jorgensen et al., 1983). The total charge of the systems was neutralized by the addition of sodium counter ions. Periodic boundary conditions were adopted.

\section{MD simulations}

130 MD simulations were performed using the PMEMD module in the Amber 20 package (Case et al., 2020). The geometry of each system was optimized (energy minimized) using the steepest descent algorithm for 500 steps, followed by the conjugate gradient algorithm for 4,500 steps. After geometry optimization, each system was heated until the temperature $(T)$ reached $300 \mathrm{~K}$ over a period of $200 \mathrm{ps}$ in the $N V T$ ensemble, while applying a harmonic restraint of $2 \mathrm{kcal} \mathrm{mol}^{-1}$ $\AA^{-2}$ on the complexes of NA and OTV, except for the hydrogen atoms. The temperature was regulated using the weak-coupling algorithm (Berendsen, Postma \& Funsteren, 1984). After 
137 heating, $10 \mathrm{~ns}$ of MD simulations were performed to equilibrate the system in the $N p T$ ensemble

138 at $T=300 \mathrm{~K}$ and a pressure $(p)$ of $1.0 \mathrm{~atm}$. The pressure was maintained using a Berendsen

139 barostat. After equilibration, additional 10-ns MD simulations were performed in the $N p T$

140 ensemble at $T=300 \mathrm{~K}$ and $p=1.0 \mathrm{~atm}$. During MD simulations, all covalent bond lengths were

141 constrained using the SHAKE algorithm (Ryckaert, Ciccotti, \& Berendsen, 1977). The time step

142 of MD simulations was set to 2 fs. A cutoff for the non-bonded intermolecular interactions was

143 set to $8 \AA$. Long-range electrostatic interactions were treated using the particle-mesh Ewald

144 method (Darden, York, \& Pedersen, 1993). Finally, four copied MD simulations were performed

145 for $100 \mathrm{~ns}$ starting with different coordinates and velocities in the $N p T$ ensemble at $T=300 \mathrm{~K}$

146 and $p=1.0 \mathrm{~atm}$, where the initial coordinates were randomly selected from the additional 10-ns

147 trajectories after equilibration and the initial velocities were randomly reassigned. The

148 production phase to be analyzed was the last $80 \mathrm{~ns}$ of MD simulations, which was determined

149 based on the root mean square displacement (RMSD) of the backbone atoms in the proteins with

150 respect to the initial structure along the simulation time. The time series of RMSD and radius of

151 gyration for the backbone atoms in the WT and I117V mutant NA are shown in Figures S1 and

152 S2. The changes in RMSD were almost constant after $20 \mathrm{~ns}$, indicating that the MD simulations

153 properly converged in the region of 20-100 ns.

154

155

\section{Results}

\section{Binding free energy calculations}

Binding free energies were determined for 400 frames extracted from the four distinctive production phases of the MD simulations, based on the Molecular Mechanics Poisson Boltzmann Surface Area (MM-PBSA) continuum solvation method (Kollman et al., 2000). The MM-PBSA calculations were performed using the MMPBSA.py program in the Amber 20 package (Miller et al., 2012; Case et al., 2020). The adaptive Poisson Boltzmann (PB) solver was used to estimate the electrostatic contribution to the solvation free energy (Baker et al., 2001). The linear PB equation was solved using a maximum of 1,000 iterations. The surface area for the nonpolar solvation energy term was determined using the Linear Combination of Pairwise Overlap (LCPO) algorithm (Weiser et al., 1999). In calculations using continuum methods, the dielectric properties of the protein interior and solvent are represented in terms of the dielectric constants. In this study, the dielectric constant of the protein interior was set to 4 , as a relatively large dielectric constant is desirable for NA, considering that its binding site contains many charged residues (Hou et al., 2011). The dielectric constant of the solvent phase was set to 80 . The ionic strength was set at $150 \mathrm{mM}$. The ratio between the longest dimension of the rectangular finitedifference grid and that of the solute was set to four.

Entropies due to the vibrational degrees of freedom were calculated for 100 configurations by normal mode analysis using the NAB program in the Amber 20 package (Case et al., 2020). The geometry of each configuration was optimized (energy minimized) with a generalized Born solvent model, using a maximum of 10,000 steps with a target root-meansquare gradient of $10^{-3} \mathrm{kcal} \mathrm{mol}^{-1} \AA^{-1}$.

\section{Binding structures and energies}

Figure 1 shows the snapshot images obtained from the MD simulations for the WT and I117V mutant NA-OTV complexes, which show the OTV binding site and the region adjacent to residue 117. As shown in Fig. 1, OTV bound to the WT or I117V mutant NA by forming hydrogen bonds with two negatively charged residues, E119 and D151, and three positively 
183 charged residues, R152, R292, and R371. Residue R118 has a positively charged side chain 184 similar to R292 and R371 in the binding site, but no hydrogen bond formation with OTV was 185 observed. This is supported by the co-crystal structure of WT A/H5N1 NA with OTV (PDB 186 code: 2HU4) (Russell et al., 2006) showing that R118 is not in a position to form hydrogen 187 bonds with OTV.

188 Table 1 summarizes the computed binding free energies $(\Delta G)$ of OTV for the WT and 189 I117V mutant NA obtained from the MM-PBSA calculations, along with the enthalpy $(\Delta H)$ and entropy $(T \Delta S)$. The binding free energies of OTV were computed to be -14.60 and $-11.88 \mathrm{kcal}$ $\mathrm{mol}^{-1}$ for the WT and I117V mutant NA, respectively. The $2.72 \mathrm{kcal} \mathrm{mol}^{-1}$ increase in the binding free energy of OTV due to the I117V mutation could slightly reduce the susceptibility of this inhibitor to NA. This is supported by the fact that the I117V mutant NA has a 3- to approximately 50-fold decrease in the relative susceptibility to OTV compared with the WT NA in H5N1 viruses (Hurt et al., 2007; McKimm-Breschkin et al., 2007; Chen et al., 2010; Ilyushina et al., 2010; McKimm-Breschkin et al., 2013; Takano et al., 2013; Creanga et al., 2017; Kode et al., 2019). According to the WHO's antiviral working group criteria, influenza A viruses with < 10 -fold change in the half maximal inhibitory concentration $\left(\mathrm{IC}_{50}\right)$ value were characterized as exhibiting normal inhibition, while those with 10- to 100 -fold and $>100$-fold changes exhibited reduced and highly reduced inhibition, respectively $(W H O, 2012)$. If experiments are done under the same conditions, the relative binding free energy of $\Delta \Delta G=\Delta G^{(1)}-\Delta G^{(2)}$ can be approximated using $\Delta \Delta G \cong R T \ln \left(\mathrm{IC}_{50}{ }^{(1)} / \mathrm{IC}_{50}{ }^{(2)}\right)$, where $R$ is the ideal gas constant and $T$ is the temperature. The experimentally observed 3 - to 50 -fold change in the $\mathrm{IC}_{50}$ value after $\mathrm{I} 117 \mathrm{~V}$ mutation corresponds to a binding free energy difference of $0.7-2.3 \mathrm{kcal} \mathrm{mol}^{-1}$. The current results are qualitatively consistent with the experimental studies, indicating that the MD simulations, which form the basis for subsequent analyses, are reliable.

In this study, we adopted the single-trajectory approach in the MM-PBSA calculation, because it assumes that no significant conformational changes occur upon ligand binding. The single-trajectory MM-PBSA approach has been widely used in previous studies, to determine binding free energy differences because of its good balance between computational cost and reliability (Wang et al., 2019). In some cases the single-trajectory approach used in this study is less reliable for determining binding free energies than the multiple-trajectory approach that accounts for conformational changes upon drug binding. However, we emphasize that the binding free energy difference of $2.72 \mathrm{kcal} \mathrm{mol}^{-1}$ between the WT and I117V mutant NA determined in the present study is in good agreement with the experimentally determined value of $0.7-2.3 \mathrm{kcal} \mathrm{mol}^{-1}$, which reveals that our MD simulations are sufficiently reliable for analyzing changes in various intra-protein interactions, such as hydrogen bonding, and secondary structures, which is the focus of this study.

As shown in Fig. 1, residue 117 did not interact directly with OTV in either WT or I117V mutant NA. Thus, the decrease in binding affinity between OTV and NA due to the I117V mutation could be the result of indirect effects due to changes in the interaction network of amino acid residues inside the protein. As shown in Table 1, the change in the entropic component $(T \Delta S)$ upon I117V mutation is almost zero, which indicates that the difference in the binding free energies $(\Delta \Delta G)$ is mostly enthalpy-driven rather than entropy-driven. Based on this observation, further analyses that focus on the factors responsible for changes in the direct interactions between OTV and NA are expected to be helpful. To elucidate the molecular mechanism by which the $1117 \mathrm{~V}$ mutation of NA at a point outside its drug-binding site could 
228

229

230

231

232

233

234

235

236

237

238

239

240

241

242

243

244

245

246

247

248

249

250

251

252

253

254

255

256

257

258

259

260

261

262

263

264

265

266

267

268

269

270

271

272

273

reduce the susceptibility to OTV, we performed the following detailed analysis based on the results of MD simulations.

\section{Hydrogen bond analysis}

Figure 2 shows the hydrogen bond occupancies of OTV for the WT and I117V mutant NAs during the MD simulations. The standard errors in hydrogen bond occupancies are small (less than $1 \%$ ); the $95 \%$ confidence intervals for hydrogen bond occupancies are shown as error bars in Fig. 2. Hydrogen bonds were assigned using PYTRAJ (Nguyen et al., 2016), a Python frontend package of the CPPTRAJ program (Roe \& Cheatham, 2013). As shown in Fig. 2, OTV bound to NA by forming hydrogen bonds with five charged amino acid residues, E119, D151, R152, R292, and R371. In hydrogen bonding with OTV, the side chains of E119 and D151 acted as hydrogen acceptors, while the side chains of R152, R292, and R371 acted as hydrogen donors. The occupancies of the hydrogen bonds formed by R292 and R371 with OTV were almost $100 \%$ in both the WT and I117V mutant NA-OTV complexes, indicating that the interactions were extremely stable. The hydrogen bond formed between OTV and R 152 was found to be relatively unstable, with an occupancy of approximately $60 \%$ in both WT and I117V mutant cases. Notable changes caused by the I117V mutation in NA were observed in the hydrogen bonds formed by E119 and D151 with OTV. Because of the I117V mutation in NA, the hydrogen bond occupancy of the E119-OTV pair increased by approximately $10 \%$, whereas the hydrogen bond occupancy of the D151-OTV pair decreased by approximately $30 \%$. D151 formed hydrogen bonds with the adjacent positively charged R156 amino acid residue when not bound to OTV. Thus, the instability of the hydrogen bond with D151 after the I117V mutation might be the major reason for the reduced binding affinity of NA to OTV.

\section{Secondary structure analysis}

Figures $3 \mathrm{~A}$ and $3 \mathrm{~B}$ show the secondary structure occupancies in the region containing the 100th to 150 th residues of NA for the WT and I117V mutant NA-OTV complexes, respectively. Figure $3 \mathrm{C}$ shows the changes in secondary structure occupancy after the I117V mutation. The secondary structure occupancies for all the residues in the NA are shown in Figure S3. The standard errors for secondary structure occupancies are small (less than 1\%); the $95 \%$ confidence intervals for secondary structure occupancies are shown as error bars in Figs. 3A, 3B and 3C. The secondary structures were classified into three simplified categories (helix, sheet, and coil) using the PYTRAJ package (Nguyen et al., 2016) based on the DSSP program (Kabsch \& Sander, 1983). The occupancies of secondary structures were calculated based on the assignment results for 3,200 three-dimensional structures extracted from four distinctive 80-ns trajectories in the production phase of MD simulations. In Fig. 3, the helix and sheet components are represented by red and blue bars, respectively, while the rest correspond to the coil.

As shown in Fig. 1, NA has an overall $\beta$-sheet-rich structure with partial helices. In the WT NA, as shown in Fig. 3A, the helix moieties were found in the region from the 105th to 111 th residues and from the 143rd to 149th residues of NA. The 117th residue of interest in this study was located in the $\beta$-sheet region formed by residues between the 115th and 124th residues. After the I117V mutation, the secondary structure of the NA was significantly altered.

As shown in Fig. 3C, the occupancy of the helix component was significantly reduced in the region between residues the 147th and 149th residues with the I117V mutation. The secondary structure near the mutation site was also changed due to the I117V mutation, indicating that the $\beta$-sheet occupancy of R118 was reduced by approximately $12 \%$. This may be

Peer] Phy. Chem. reviewing PDF | (PCHEM-2021:06:62231:2:0:NEW 20 Oct 2021) 
274 due to a change in the orientation of R118 caused by the mutation of the bulkier Ile to the smaller

275 Val at the 117th residue, which reduces the hydrogen-bonding interaction with residue L134

276 located in the adjacent antiparallel $\beta$-sheet moiety. Such conformational changes of R118 at a

277 point inside the drug-binding site of NA would lead to a decrease in the binding affinity of the

278 I117V mutant for OTV, due to the indirect effect of the Ile-to-Val mutation at residue 117.

279

280

281

282

283

284

285

286

287

288

289

290

291

292

293

294

295

296

297

298

299

300

301

302

303

304

305

306

307

308

309

310

311

312

\section{Discussion}

314 WT NA-OTV complex

315 In the WT NA-OTV complex, the residue-residue interaction between R118 and E119 may play

316 a key role in enhancing the residue-drug interaction between D151 and OTV to increase the

317 binding affinity of the WT NA to OTV. As shown in Figs. 1A and 5A, the negatively charged

318 E119 interacts with the positively charged amino group of OTV, together with the negatively

319 charged D151. Here, E119 and D151 tend to approach each other when interacting with OTV 
320

321

322

323

324

325

326

327

328

329

330

331

332

333

334

335

336

337

338

339

340

341

342

343

344

345

346

347

348

349

350

351

352

353

354

355

356

357

358

359

360

361

362

363

364

365

simultaneously, but the closer they are, the stronger the electrostatic repulsion between the negatively charged side chains. However, as shown in Fig. 2, the hydrogen bond occupancy of the E119-OTV and D151-OTV pairs was approximately $80 \%$ for both, indicating that E119 and D151 can form relatively stable hydrogen bonds with OTV. As shown in Fig. 3A, R118 is in the $\beta$-sheet region, indicating that its side chain can be rigidly oriented. Owing to the strong directivity of R118, derived from its secondary structure formation, its positively charged side chain can frequently interact in parallel with the negatively charged side chain of the adjacent E119. When R118 and E119 interact, the positive and negative charges of their side chains neutralize each other, thereby suppressing the electrostatic repulsion between E119 and D151. Thus, both E119 and D151 can simultaneously form hydrogen-bonding interactions with OTV, which contributes to the enhancement of the binding affinity of NA to OTV.

\section{I117V mutant NA-OTV complex}

In the I117V mutant NA-OTV complex, the binding affinity of NA to OTV may be reduced by the weakening of the residue-drug interaction between D151 and OTV, accompanied by a decrease in the opportunity for residue-residue interaction between R118 and E119. As shown in Fig. 3C, the occupancy of R118 forming the $\beta$-sheet structure decreased after the I117V mutation, indicating that the directionality of its side chain was weakened. The weakening of the directionality of its positively charged side chain reduces the opportunity for interaction with the negatively charged side chain of the adjacent E119. The reduced interactions between the side chains of R118 and E119 are shown in Fig. 4. As mentioned earlier, in the WT NA-OTV complex, the interaction between R118 and E119 can contribute to reducing the electrostatic repulsion between E119 and D151. However, in the I117V mutant NA-OTV complex, the electrostatic repulsion between E119 and D151 can be enhanced, since R118 has less opportunity for interaction with E119. This inhibits both E119 and D151 from simultaneously forming hydrogen-bonding interactions with the same positively charged amino group of OTV, resulting in a decrease in the binding affinity between NA and OTV. Thus, the change in the interactions of these residues after the I117V mutation slightly reduces the binding affinity of NA to OTV, resulting in a reduction in OTV drug susceptibility to influenza viruses.

As mentioned in the Introduction, several computational studies that use MD simulations have reported on the molecular mechanism associated with the lower susceptibility of the $1117 \mathrm{~V}$ mutant to OTV (Takano et al., 2013; Mhlongo \& Soliman, 2015). Takano et al. (2013) analyzed a single 2.5-ns MD trajectory to show that the loss of hydrogen bonding between the R118 side chain in NA and the OTV carboxyl group after I1 $17 \mathrm{~V}$ mutation is responsible for the reduced susceptibility of NA to OTV. A previous study by Takano et al. (2013) showed that hydrogen bonds are formed between the R118 residue of WT NA and OTV; however this was not observed in the previous study by Mhlongo and Soliman (2015) or in the current study. We speculate that this discrepancy is due the trajectory used for analysis in the previous study by Takano et al. (2013), which was too short to adequately sample the conformational space of the system. Mhlongo and Soliman (2015) analyzed four distinctive 25-ns MD trajectories and suggested that the I117V mutation affects residue-residue interactions in NA that cause the drugbinding site to change its conformation, thereby altering residue-drug interactions between NA and OTV; however, the details were not clear. In the current study, we elucidated correlations between the residue-residue interaction of the R118-E119 pair and the residue-drug interaction of the D151-OTV pair in NA-OTV complexes by analyzing four distinctive 80 -ns trajectories obtained from MD simulations, as shown in Fig. 4. As a result, we clarified the detailed

Peer] Phy. Chem. reviewing PDF | (PCHEM-2021:06:62231:2:0:NEW 20 Oct 2021) 
378

379

380

381

382

383

384

385

386

387

388

389

390

391

392

393

394

395

396

397

398

399

400

401

402

403

404

405

406

407

408

409

\section{Conclusions}

molecular mechanism by which the I117V mutation in NA alters the inter-residue interactions between R118, E119, and D151, and destabilizes the residue-drug interaction between D151 and OTV, thereby reducing the susceptibility of NA to OTV.

We speculate that the I117V mutation not only affects the susceptibility of NA to OTV, but also viral fitness. With regard to viral fitness, we expect to extend the present study in the future to clarify the effects of the $1117 \mathrm{~V}$ mutation on the binding affinity of the natural sialic acid substrate to NA. However, according to Adams et al. (2019), viral fitness not only depends on the binding affinity between the substrate and the enzyme, but also on the catalytic efficiency of the enzyme. Hence, clarifying the catalytic reaction mechanism of NA using expensive computational methods, such as the QM/MM method (Sousa et al., 2017), is required to study the effect of NA mutations on viral fitness. Since analyzing the catalytic reaction of NA is far beyond the scope of this study, we simply mention it here as a future subject.

\section{Designing a potential drug design against $1117 \mathrm{~V}$ mutant strains}

As summarized in Table 1, the I117V mutation in NA reduces the binding free energy of NA to OTV by $2.72 \mathrm{kcal} \mathrm{mol}^{-1}$, which corresponds to an approximate 100 -fold decrease in the relative susceptibility of the I117V mutant NA to OTV compared to that of WT NA. The $\mathrm{IC}_{50}$ value of OTV has been experimentally observed to change by a factor of 3-50 upon $1117 \mathrm{~V}$ mutation in NA (Hurt et al., 2007; McKimm-Breschkin et al., 2007; Chen et al., 2010; Ilyushina et al., 2010; McKimm-Breschkin et al., 2013; Takano et al., 2013; Creanga et al., 2017; Kode et al., 2019). Compared with substitutions that are selected under drug pressure and increase $\mathrm{IC}_{50}$ by more than 600 -fold, such as $\mathrm{H} 274 \mathrm{Y}$ (Hurt et al., 2012), the I117V mutation does not dramatically affect susceptibility to OTV. Therefore, OTV treatment may be effective against the I117V mutant strain of the influenza virus. However, the I117V mutation may affect OTV resistance in synergism with other mutations. For example, Hurt et al. (2012) found that the introduction of the dual I117V + H274Y mutation in NA significantly decreased susceptibility to OTV (a 1896-fold increase in $\mathrm{IC}_{50}$ ) compared to that resulting from the $\mathrm{H} 274 \mathrm{Y}$ mutation alone (a 650-fold increase in $\mathrm{IC}_{50}$ ). Therefore, based on the new knowledge gained in this study, we propose guidelines for drug design that avoid the loss of drug sensitivity associated with the I117V mutation in preparation for the possible emergence of potent drug-resistant strains.

Based on our study, we suggest that an inhibitor with a longer positively charged group is better than one with a shorter positively charged group, such as the amino group in OTV, to avoid resistance from the I117V mutation that affects interactions between the inhibitor and the E119 and D151 NA binding site residues. A longer positively charged group in the inhibitor helps to reduce electrostatic repulsion between the negatively charged E119 and D151 side chains. For example, OTV has a short positively charged amino group that interacts with residues E119 and D151, while zanamivir has a long positively charged guanidino group. In fact, the $\mathrm{I} 117 \mathrm{~V}$ mutation in NA resulted in a significant 50 -fold change in the $\mathrm{IC}_{50}$ value for OTV but only a 1.6-fold change in the $\mathrm{IC}_{50}$ value for zanamivir, which indicates that zanamivir is effective against the I117V mutant strain (McKimm-Breschkin et al., 2013). In this study, we used molecular simulations to understand the molecular mechanism of OTV resistance associated with the I117V mutation in NA in detail, which led to the establishment of new molecular design guidelines that effectively solve the drug resistance problem.

Peer] Phy. Chem. reviewing PDF | (PCHEM-2021:06:62231:2:0:NEW 20 Oct 2021) 
411 In this study, we theoretically investigated the molecular mechanism of reduced OTV drug

412 susceptibility in the A/H5N1 influenza virus harboring the NA I117V mutation using MD

413 simulations.

414 In the WT NA-OTV complex, the interaction between R118 and E119 can play an 415 important role in increasing the binding affinity of NA to OTV. In this case, the positively 416 charged side chain of R118, located in the $\beta$-sheet region, can frequently interact with the 417 negatively charged side chain of E119, preventing the electrostatic repulsion between E119 and 418 D151. This enables both the negatively charged side chains of E119 and D151 to simultaneously 419 form hydrogen bonding interactions with the positively charged amino group of OTV, thereby 420 contributing significantly to the binding affinity between NA and OTV.

In the I117V mutant NA-OTV complex, the binding affinity of NA to OTV can be reduced by decreasing the opportunity for interaction between R118 and E119. In this case, the mutation reduces the tendency of R118 to form the $\beta$-sheet structure, leading to less frequent interaction between its positively charged side chain and the negatively charged side chain of E119. This increases the electrostatic repulsion between E119 and D151, making it difficult for both to simultaneously form hydrogen bonds with OTV, which in turn reduces the binding affinity between NA and OTV. Thus, after the I117V mutation in NA, influenza viruses are less susceptible to OTV because of changes in the residue interactions between R118, E119, and D151.

The present study has successfully clarified the molecular mechanism by which the I1 17V mutation in NA reduces the OTV drug susceptibility of the A/H5N1 influenza virus.

\section{References}

Adams SE, Lee N, Lugovtsev VY, Kan A, Donnelly RP, Ilyushina NA. 2019. Effect of influenza H1N1 neuraminidase V116A and I117V mutations on NA activity and sensitivity to NA inhibitors. Antiviral Research 169:104539 DOI: 10.1016/j.antiviral.2019.104539

Baker NA, Sept D, Joseph S, Holst MJ, McCammon JA. 2001. Electrostatics of nanosystems: application to microtubules and the ribosome. Proceedings of the National Academy of Sciences of the United States of America 98:10037-10041 DOI: 10.1073/pnas.181342398

Bayly CI, Cieplak P, Cornell WD, Kollman PA. 1993. A well-behaved electrostatic potential based method using charge restraints for determining atom-centered charges: the RESP model. Journal of Physical Chemistry 97:10269-10280 DOI: 10.1021/j100142a004

Berendsen HJC, Postma JPM, Funsteren WF. 1984. Molecular dynamics with coupling to an external bath. Journal of Chemical Physics 81:3684-3690 DOI: 10.1063/1.448118

Case DA, Belfon K, Ben-Shalom IY, Brozell SR, Cerutti DS, Cheatham III TE, Cruzeiro VWD, Darden TA, Duke RE, Giambasu G, Gilson MK, Gohlke H, Goetz AW, Harris R, Izadi S, Izmailov SA, Kasavajhala K, Kovalenko A, Krasny R, Kurtzman T, Lee TS, LeGrand S, Li P, Lin C, Liu J, Luchko T, Luo R, Man V, Merz KM, Miao Y, Mikhailovskii O, Monard G, Nguyen H, Onufriev A, Pan F, Pantano S, Qi R, Roe DR, Roitberg A, Sagui 455 C, Schott-Verdugo S, Shen J, Simmerling CL, Skrynnikov NR, Smith J, Swails J, Walker 
463

464

465

466

467

468

469

470

471

472

473

474

475

476

477

478

479

480

481

482

483

484

485

486

487

488

489

490

491

492

493

494

495

496

497

498

499

500

501

RC, Wang J, Wilson L, Wolf RM, Wu X, Xiong Y, Xue Y, York DM, Kollman PA. 2020. In: $A M B E R$ 2020. San Francisco: University of California.

Chen Z, Zheng YH, Lin Y, Collins PJ, Hay AJ. 2010. Impact of avian influenza virus H5N1 neuraminidase mutations on the activity of neuraminidase and the sensibility to neuraminidase inhibitors. National Medical Journal of China 90:1924-1928 DOI: 10.3760/cma.j.issn.03762491.2010.27.014

Collins PJ, Haire LF, Lin YP, Liu J, Russell RJ, Walker PA, Skehel JJ, Martin SR, Hay AJ, Gamblin SJ. 2008. Crystal structures of oseltamivir-resistant influenza virus neuraminidase mutants. Nature 453:1258-1261 DOI: 10.1038/nature06956

Colman PM, Hoyne PA, Lawrence MC. 1993. Sequence and structure alignment of paramyxovirus hemagglutinin-neuraminidase with influenza virus neuraminidase. Journal of virology 67(6):2972-2980 DOI: 10.1128/JVI.67.6.2972-2980.1993

Colman PM, Varghese JN, Laver WG. 1983. Structure of the catalytic and antigenic sites in influenza virus neuraminidase. Nature 303(5912):41-44 DOI: 10.1038/303041a0

Creanga A, Hang NLK, Cuong VD, Nguyen HT, Phuong HVM, Thanh LH, Thach NC, Hien PT, Tung N, Jang Y, Balish A, Dang NH, Duong MT, Huong NT, Hoa DN, Tho ND, Klimov A, Kapella BK, Gubareva L, Kile JC, Hien NT, Mai LQ, Davis CT. 2017. Highly pathogenic avian influenza A (H5N1) viruses at the animal-human interface in Vietnam, 20032010. The Journal of Infectious Diseases 216:S529-S538 DOI: 10.1093/infdis/jix003

Darden T, York D, Pedersen L. 1993. Particle mesh Ewald: an $\mathrm{N} \log (\mathrm{N})$ method for Ewald sums in large systems. Journal of Chemical Physics 98:10089-10092 DOI: 10.1063/1.464397

Dolinsky TJ, Nielsen JE, McCammon JA, Baker NA. 2004. PDB2PQR: an automated pipeline for the setup, execution, and analysis of Poisson Boltzmann electrostatics calculations. Nucleic Acids Research 32:W665-W667 DOI: 10.1093/nar/gkh381

Fouchier RA, Munster V, Wallensten A, Bestebroer TM, Herfst S, Smith D, Rimmelzwaan GF, Olsen B, Osterhaus AD. 2005. Characterization of a novel influenza A virus hemagglutinin subtype (H16) obtained from black-headed gulls. Journal of Virology 79:2814-2822 DOI: 10.1128/JVI.79.5.2814-2822.2005

Frisch MJ, Trucks GW, Schlegel HB, Scuseria GE, Robb MA, Cheeseman JR, Scalmani G, Barone V, Petersson GA, Nakatsuji H, Li X, Caricato M, Marenich AV, Bloino J, Janesko BG, Gomperts R, Mennucci B, Hratchian HP, Ortiz JV, Izmaylov AF, Sonnenberg JL, Williams-Young D, Ding F, Lipparini F, Egidi F, Goings J, Peng B, Petrone A, Henderson T, Ranasinghe D, Zakrzewski VG, Gao J, Rega N, Zheng G, Liang W, Hada M, Ehara M, Toyota K, Fukuda R, Hasegawa J, Ishida M, Nakajima T, Honda Y, Kitao O, Nakai H, Vreven T, Throssell K, Montgomery Jr JA, Peralta JE, Ogliaro F, Bearpark MJ, Heyd JJ, Brothers EN, Kudin KN, Staroverov VN, Keith TA, Kobayashi R, Normand J, Raghavachari K, Rendell AP, Burant JC, Iyengar SS, Tomasi J, Cossi M, Millam JM,

Peer) Phy. Chem. reviewing PDF | (PCHEM-2021:06:62231:2:0:NEW 20 Oct 2021) 
502 Klene M, Adamo C, Cammi R, Ochterski JW, Martin RL, Morokuma K, Farkas O, 503 Foresman JB, Fox DJ. 2016. Gaussian 16, Revision C.01. Wallingford CT: Gaussian, Inc.

504

505

506

507

508

509

510

511

512

513

514

515

516

517

518

519

520

521

522

523

524

525

526

527

528

529

530

531

532

533

534

535

536

537

538

539

540

541

542

543

544

545

546

547
Gamblin SJ, Skehel JJ. 2010. Influenza hemagglutinin and neuraminidase membrane glycoproteins. Journal of Biological Chemistry 285:28403-28409 DOI: 10.1074/jbc.r110.129809

Govorkova EA, Ilyushina NA, McClaren JL, Naipospos TS, Douangngeun B, Webster RG. 2009. Susceptibility of highly pathogenic H5N1 influenza viruses to the neuraminidase inhibitor oseltamivir differs in vitro and in a mouse model. Antimicrobial agents and chemotherapy 53(7):3088-3096 DOI: 10.1128/AAC.01667-08

Han N, Liu X, Mu Y. 2012. Exploring the mechanism of zanamivir resistance in a neuraminidase mutant: a molecular dynamics study. PLoS ONE 7(9):e44057 DOI: 10.1371/journal.pone.0044057

Hou T, Wang J, Li Y, Wang W. 2011. Assessing the performance of the MM/PBSA and MM/GBSA Methods. 1. The accuracy of binding free energy calculations based on molecular dynamics simulations. Journal of Chemical Information and Modeling 51:69-82 DOI: $10.1021 /$ ci100275a

Hurt AC, Selleck P, Komadina N, Shaw R, Brown L, Barr IG. 2007. Susceptibility of highly pathogenic $\mathrm{A}(\mathrm{H} 5 \mathrm{~N} 1)$ avian influenza viruses to the neuraminidase inhibitors and adamantanes. Antiviral Research 73:228-231 DOI: 10.1016/j.antiviral.2006.10.004

Hurt AC, Leang SK, Speers DJ, Barr IG, Maurer-Stroh S. 2012. Mutations I117V and I1 17M and oseltamivir sensitivity of pandemic (H1N1) 2009 viruses. Emerging Infectious Diseases 18:109-112 DOI: 10.3201/eid1801.111079

Ilyushina NA, Seiler JP, Rehg JE, Webster RG, Govorkova EA. 2010. Effect of neuraminidase inhibitor-resistant mutations on pathogenicity of clade $2.2 \mathrm{~A} /$ Turkey/15/06 (H5N1) influenza virus in ferrets. PLoS Pathogens 6:e1000933 DOI: 10.1371/journal.ppat. 1000933

Jorgensen WL, Chandrasekhar J, Madura JD, Impey RW, Klein ML. 1983. Comparison of simple potential functions for simulating liquid water. The Journal of Chemical Physics 79:926935 DOI: $10.1063 / 1.445869$

Kabsch W, Sander C. 1983. Dictionary of protein secondary structure: pattern recognition of hydrogen-bonded and geometrical features. Biopolymers 22:2577-2637 DOI:

10.1002/bip.360221211

Kar P, Knecht V. 2012. Mutation-induced loop opening and energetics for binding of tamiflu to influenza N8 neuraminidase. The Journal of Physical Chemistry B 116(21):6137-6149 DOI: $10.1021 / \mathrm{jp} 3022612$ 
Kim CU, Lew W, Williams MA, Liu H, Zhang L, Swaminathan S, Bischofberger N, Chen MS, Mendel DB, Tai CY, Laver WG, Stevens RC. 1997. Influenza neuraminidase inhibitors possessing a novel hydrophobic interaction in the enzyme active site: design, synthesis, and structural analysis of carbocyclic sialic acid analogs with potent anti-influenza activity. Journal

552 of the American Chemical Society 119:681-690 DOI: 10.1021/ja963036

Kode SS, Pawar SD, Tare DS, Keng SS, Hurt AC, Mullick J. 2019. A novel I117T substitution in neuraminidase of highly pathogenic avian influenza H5N1 virus conferring reduced susceptibility to oseltamivir and zanamivir. Veterinary Microbiology 235:21-24 DOI: 10.1016/j.vetmic.2019.06.005

Kollman PA, Massova I, Reyes C, Kuhn B, Huo S, Chong L, Lee M, Lee T, Duan Y, Wang W, Donini O, Cieplak P, Srinivasan J, Case DA, Cheatham TE. 2000. Calculating Structures and Free Energies of Complex Molecules: Combining Molecular Mechanics and Continuum Models. Accounts of Chemical Research 33:889-897 DOI: 10.1021/ar000033j

Maier JA, Martinez C, Kasavajhala K, Wickstrom L, Hauser KE, Simmerling C. 2015. ff14SB: Improving the accuracy of protein side chain and backbone parameters from ff99SB. Journal of Chemical Theory and Computation 11:3696-3713 DOI: 10.1021/acs.jctc.5b00255

Marinova-Petkova A, Feeroz MM, Rabiul Alam SM, Kamrul Hasan M, Akhtar S, JonesEngel L, Walker D, McClenaghan L, Rubrum A, Franks J, Seiler P, Jeevan T, McKenzie P, Krauss S, Webby RJ, Webster RG. 2014. Multiple introductions of highly pathogenic avian influenza H5N1 viruses into Bangladesh. Emerging microbes \& infections 3:e11 DOI: $10.1038 / \mathrm{emi} .2014 .11$

McKimm-Breschkin JL, Barrett S, Pudjiatmoko, Azhar M, Wong FY, Selleck P, Mohr PG, McGrane J, Kim M. 2013. I222 neuraminidase mutations further reduce oseltamivir susceptibility of Indonesian clade 2.1 highly pathogenic avian influenza A (H5N1) viruses. PLoS One 8:e66105 DOI: 10.1371/journal.pone.0066105

McKimm-Breschkin JL, Selleck PW, Usman TB, Johnson MA. 2007. Reduced sensitivity of influenza A (H5N1) to oseltamivir. Emerging Infectious Diseases 13:1354-1357 DOI: 10.3201/eid1309.07-0164

McKimm-Breschkin JL. 2012. Influenza neuraminidase inhibitors: antiviral action and mechanisms of resistance. Influenza and Other Respiratory Viruses 7:25-36 DOI: 10.1111/irv. 12047

Mhlongo NN, Soliman MES. 2015. Single H5N1 influenza A neuraminidase mutation develops resistance to oseltamivir due to distorted conformational and drug binding landscape: multiple molecular dynamics analyses. RSC Advances 5:10849-10861 DOI: 10.1039/c4ra13494j 
592 Miller III BR, McGee Jr TD, Swails JM, Homeyer N, Gohlke H, Roitberg AE.

593 2012. MMPBSA.py: an efficient program for end-state free energy calculations. Journal of

594 Chemical Theory and Computation 8:3314-3321 DOI: 10.1021/ct300418h

595

596

597

Monto AS, McKimm-Breschkin JL, Macken C, Hampson AW, Hay A, Klimov A, Tashiro

598

599

600

601 M, Webster RG, Aymard M, Hayden FG, Zambon M. 2006. Detection of influenza viruses resistant to neuraminidase inhibitors in global surveillance during the first 3 years of their use. Antimicrobial agents and chemotherapy 50:2395-2402 DOI: 10.1128/AAC.01339-05

Nguyen H, Roe DR, Swails J, Case DA. 2016. PYTRAJ: interactive data analysis for molecular

602

603

604 dynamics simulations. Available at https://github.com/Amber-MD/pytraj

605

606

Rameix-Welti MA, Agou F, Buchy P, Mardy S, Aubin JT, Véron M, van der Werf S, Naffakh N. 2006. Natural variation can significantly alter the sensitivity of influenza A (H5N1) viruses to oseltamivir. Antimicrobial agents and chemotherapy 50:3809-3815 DOI:

607 10.1128/AAC.00645-06

608

609

Roe DR, Cheatham III TE. 2013. PTRAJ and CPPTRAJ: software for processing and analysis

610 of molecular dynamics trajectory data. Journal of Chemical Theory and Computation 9:3084-

611

612

613 3095 DOI: $10.1021 /$ ct400341p

614

Russell RJ, Haire LF, Stevens DJ, Collins PJ, Lin YP, Blackburn GM, Hay AJ, Gamblin

615

616

617

618

619

620 SJ, Skehel JJ. 2006. The structure of H5N1 avian influenza neuraminidase suggests new opportunities for drug design. Nature 443:45-49 DOI: 10.1038/nature05114

Ryckaert JP, Ciccotti G, Berendsen HJC. 1977. Numerical integration of the cartesian equations of motion of a system with constraints: molecular dynamics of n-alkanes. Journal of Computational Physics 23:327-341 DOI: 10.1016/0021-9991(77)90098-5

623

Schaduangrat N, Phanich J, Rungrotmongkol T, Lerdsamran H, Puthavathana P, Ubol S. 2016. The significance of naturally occurring neuraminidase quasispecies of $\mathrm{H} 5 \mathrm{~N} 1$ avian influenza virus on resistance to oseltamivir: a point of concern. The Journal of general virology 97(6):1311-1323 DOI: 10.1099/jgv.0.000444

Smith BJ, Huyton T, Joosten RP, McKimm-Breschkin JL, Zhang JG, Luo CS, Lou MZ, Labrou NE, Garrett TPJ. 2006. Structure of a calcium-deficient form of influenza virus neuraminidase: implications for substrate binding. Acta Crystallographica Section D Structural Biology 62:947-952 DOI: 10.1107/S0907444906020063

630

Sousa SF, Ribeiro AJM, Neves RPP, Brás NF, Cerqueira NMFSA, Fernandes PA, Ramos MJ. 2017. Application of quantum mechanics/molecular mechanics methods in the study of enzymatic reaction mechanisms. Wiley Interdisciplinary Reviews: Computational Molecular 
638 substitution in the neuraminidase of subtype H5N1 avian influenza A viruses. The Journal of

639 Infectious Diseases 207:89-97 DOI: 10.1093/infdis/jis633

640

641 Wang E, Sun H, Wang J, Wang Z, Liu H, Zhang JZH, Hou T. 2019. End-Point Binding Free

642 Energy Calculation with MM/PBSA and MM/GBSA: Strategies and Applications in Drug

643 Design. Chemical Reviews 119:9478-9508 DOI: 10.1021/acs.chemrev.9b00055

644

645

646

647

Wang J, Wolf RM, Caldwell JW, Kollman PA, Case DA. 2004. Development and testing of a general amber force field. Journal of Computational Chemistry 25:1157-1174 DOI:

648 $10.1002 /$ jcc. 20035

650

Webster RG, Bean WJ, Gorman OT, Chambers TM, Kawaoka Y. 1992. Evolution and

651 ecology of influenza A viruses. Microbiological reviews 56:152-179 DOI: 10.1128/mr.56.1.152179.1992

652

Weiser J, Shenkin PS, Still WC. 1999. Approximate atomic surfaces from linear combinations of pairwise overlaps (LCPO). Journal of Computational Chemistry 20:217-230 DOI: 10.1002/(SICI)1096-987X(19990130)20:2<217::AID-JCC4>3.0.CO;2-A

\section{Woods CJ, Malaisree M, Pattarapongdilok N, Sompornpisut P, Hannongbua S,}

Mulholland AJ. 2012. Long time scale GPU dynamics reveal the mechanism of drug resistance of the dual mutant I223R/H275Y neuraminidase from H1N1-2009 influenza

661 virus. Biochemistry 51(21):4364-4375 DOI: 10.1021/bi300561n

World Health Organization. 2012. Meetings of the WHO working group on surveillance of influenza antiviral susceptibility - Geneva November 2011 and June 2012. Weekly Epidemiological Record 87:369-374 Available at https://apps.who.int/iris/handle/10665/241965 


\section{Figure 1}

Snapshot images of the neuraminidase-oseltamivir complexes.

(A) Wild-type (WT) and (B) I117V mutant NA-OTV complexes. The upper panels show the overall structure of the complex in the cartoon representation, where the helix and sheet parts are colored in red and blue, respectively. The lower panels show the drug-binding site and the region adjacent to residue 117, where the positively charged residues (R118, R152, R156, R292, and R371) in the OTV binding site are represented in blue, and the negatively charged residues (E119 and D151) are represented in red. 


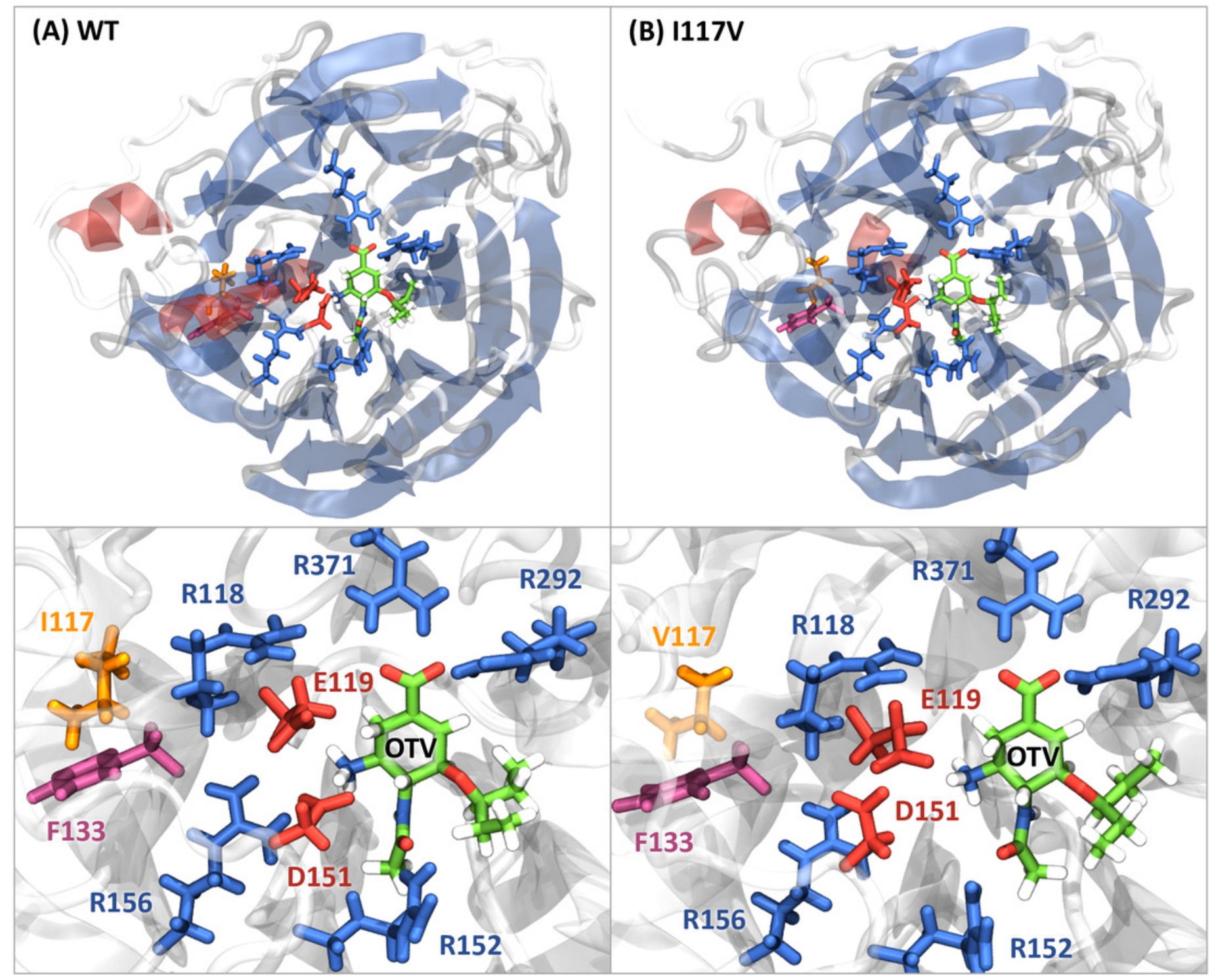




\section{Figure 2}

Hydrogen bond occupancies of oseltamivir for the wild-type (WT) and I117V mutant neuraminidases.

In hydrogen bonding with OTV, the side chains of E119 and D151 acted as hydrogen acceptors, while the side chains of R152, R292, and R371 acted as hydrogen donors. Error bars represent $95 \%$ confidence intervals.

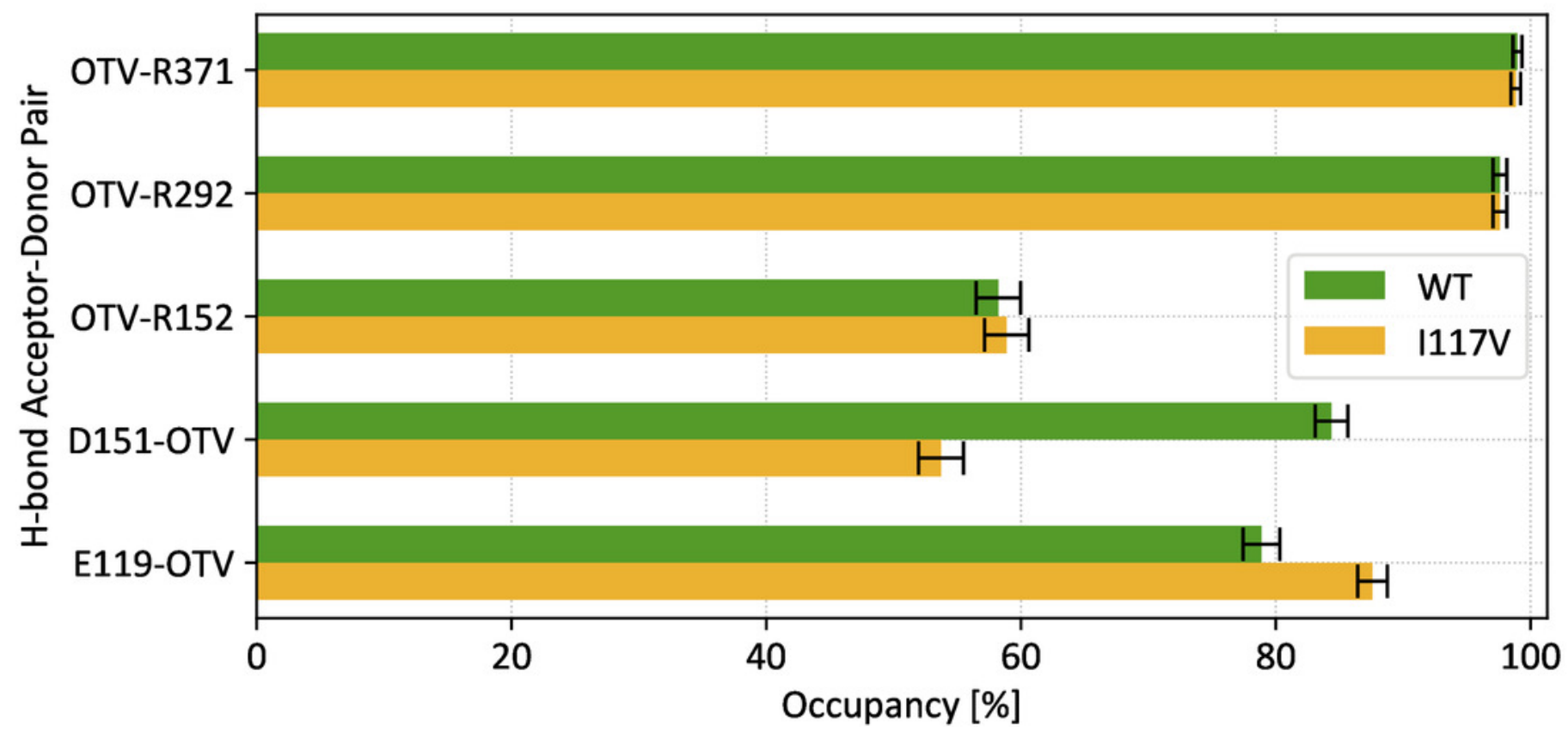


Figure 3

Secondary structure occupancies in the region containing the 100th to 150th residues for the neuraminidase-oseltamivir complexes.

(A) The wild-type (WT) and (B) I117V mutant NA-OTV complexes. (C) Change in the secondary structure occupancies due to the I117V mutation. The secondary structures were classified into three simplified categories: helix, sheet, and coil . The helix and sheet components are represented by red and blue bars, respectively, while the rest correspond to the coil. Error bars represent 95\% confidence intervals. 

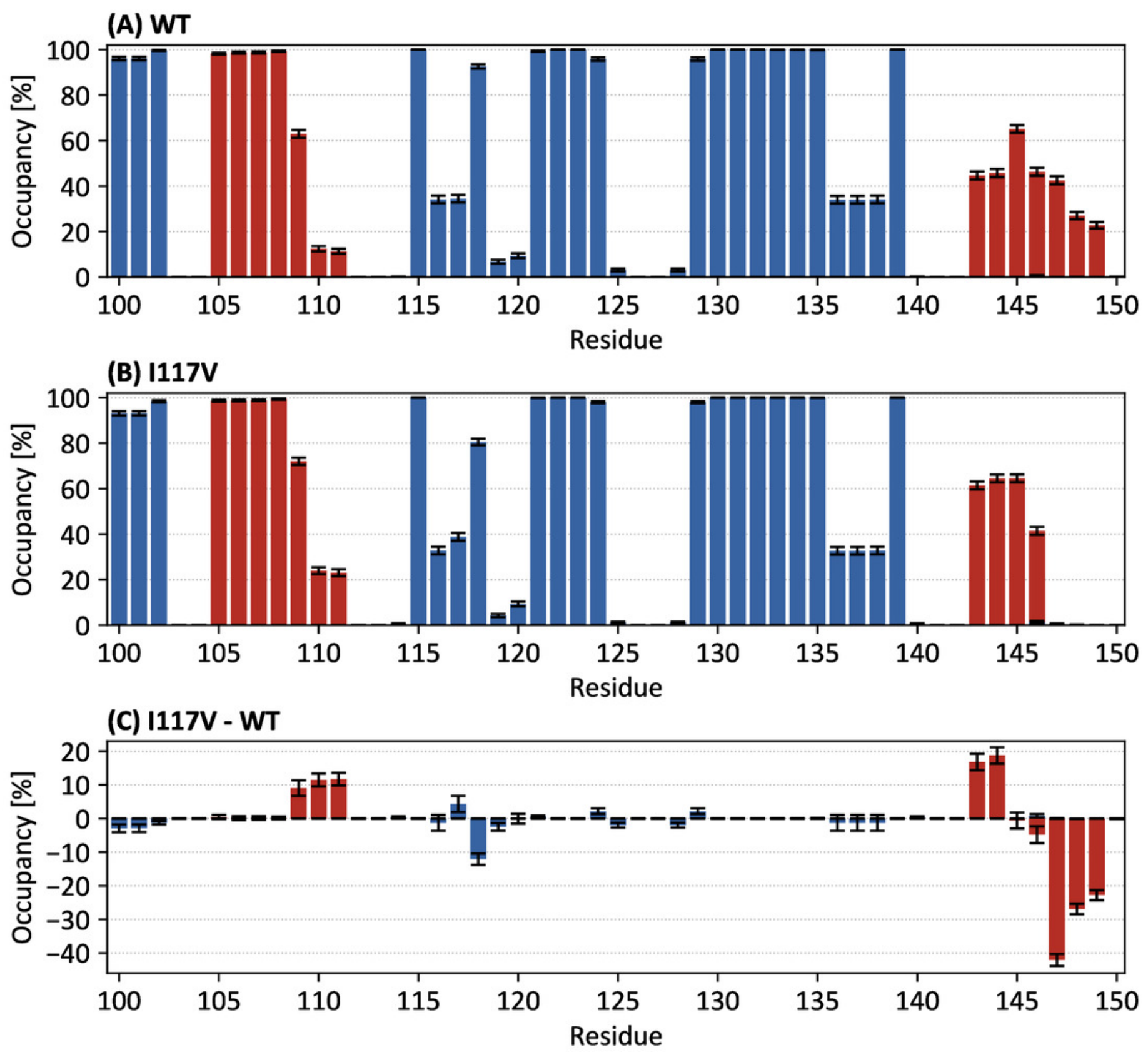


\section{Figure 4}

Correlations between residue-residue and residue-drug interactions.

Scatter plots and probability densities for the distance of the R118-E119 pair $\left(R_{\mathrm{R} 118-\mathrm{E} 119}\right)$ versuS the distance of the D151-OTV pair $\left(R_{\text {D151-orv }}\right)$ in the wild-type $(\mathrm{WT})$ and I117V mutant NA-OTV complexes. 


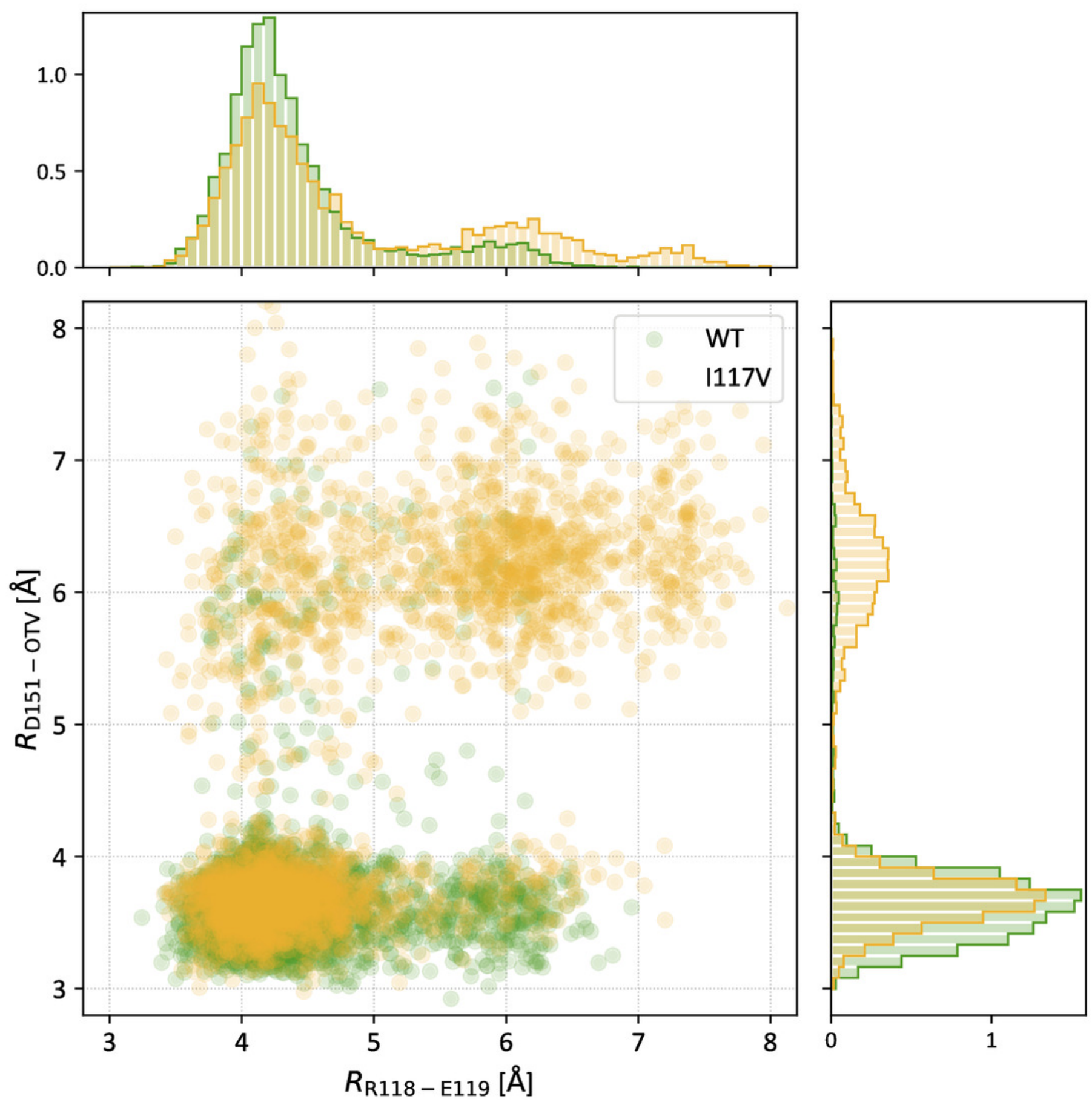




\section{Figure 5}

Superimposed snapshot images for the neuraminidase-oseltamivir complexes.

(A) Wild-type (WT) and (B) I117V mutant NA-OTV complexes. OTV is represented in green, and residue 117 is indicated in orange. The positively charged residues (R118, R152, R156, R292, and R371) in the OTV binding site are represented in blue, and the negatively charged residues (E119 and D151) are represented in red.

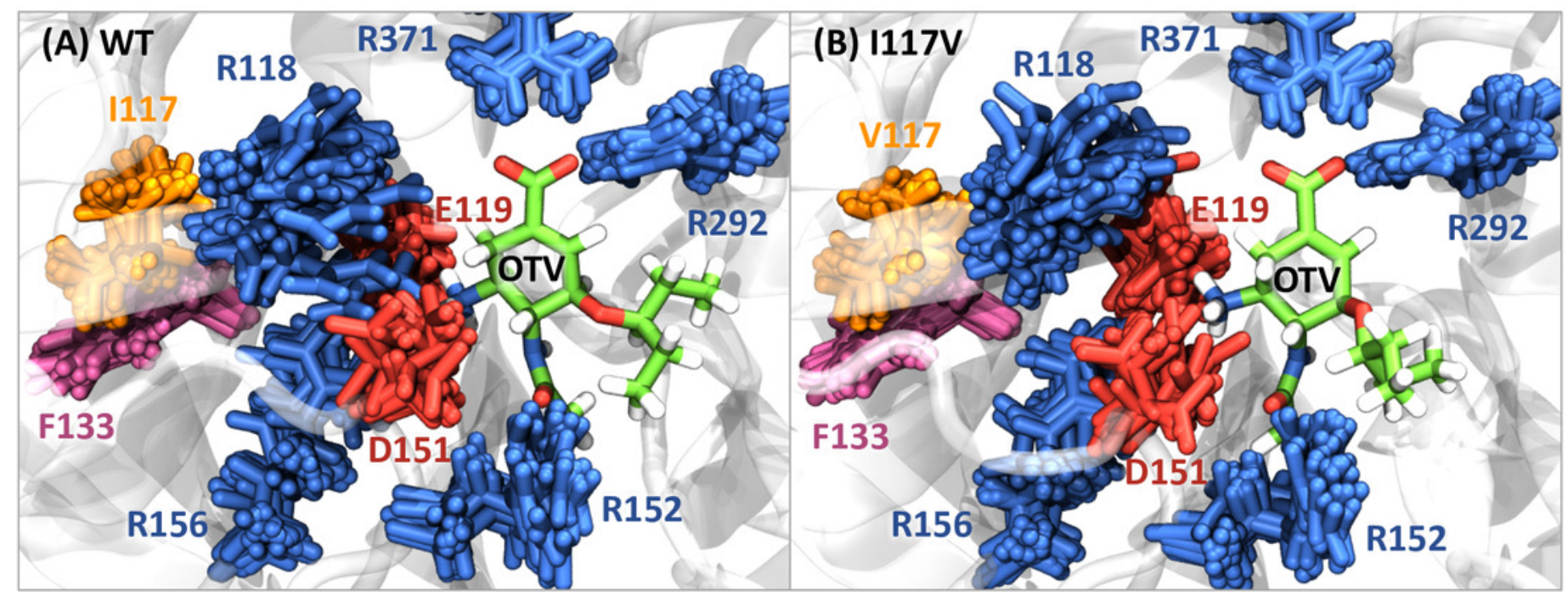




\section{Table $\mathbf{1}$ (on next page)}

Calculated binding free energies (in $\mathrm{kcal} \mathrm{mol}^{-1}$ ) for oseltamivir to the wild-type (WT) and I117V mutant influenza neuraminidase obtained from the MM-PBSA calculations. 
1

\begin{tabular}{ccccc}
\hline & $\boldsymbol{\Delta} \boldsymbol{H}$ & $\boldsymbol{T} \boldsymbol{\Delta} \boldsymbol{S}$ & $\boldsymbol{\Delta} \boldsymbol{\Delta}$ & $\boldsymbol{\Delta} \boldsymbol{G}$ \\
\hline WT & $-33.78 \pm 0.08$ & $-19.18 \pm 1.15$ & $-14.60 \pm 1.23$ & \\
$\mathrm{I117V}$ & $-31.06 \pm 0.08$ & $-19.18 \pm 1.20$ & $-11.88 \pm 1.28$ & 2.72 \\
\hline
\end{tabular}

2 